\title{
Integrated analysis of mRNA and miRNA expression profiles in pancreatic ductal adenocarcinoma
}

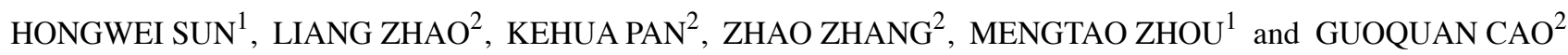 \\ Departments of ${ }^{1}$ Hepatobiliary Surgery and ${ }^{2}$ Radiology, The First Affiliated Hospital of Wenzhou Medical University, \\ Wenzhou, Zhejiang, P.R. China
}

Received October 27, 2016; Accepted December 1, 2016

DOI: 10.3892/or.2017.5526

\begin{abstract}
In the present study, to investigate the potential molecular mechanism of pancreatic ductal adenocarcinoma (PDAC), mRNA and miRNA expression profiles were integrated for systematic analysis. Results showed that a total of 76 common differentially expressed genes (DEGs) were identified from 2 mRNA expression profiles that contained 39 tumor and 15 normal samples. Notably, the tumor and normal samples were able to be clearly classified into 4 groups based on the DEGs. mRNA-miRNA regulation network analysis indicated that 22 out of the 76 DEGs including MUC4, RRM2 and CCL2 are regulated by 5 reported miRNAs. Survival analysis using SurvExpress database demonstrated that the common DEGs were able to significantly differentiate low- and high-risk PDAC groups in 4 datasets. In summary, various biological processes are probably involved in the development and progression of PDAC. Firstly, activation of MUC4 induces nuclear translocation of $\beta$-catenin and promotes the process of angiogenesis that provides necessary nutrition or oxygen for cancer cells. Then, RRM2 induces the invasiveness of PDAC via $N F-\kappa B$. Finally, the formation of an immunosuppressive tumor microenvironment by recruiting regulatory $\mathrm{T}$ cells with high expression of CCL2 further promotes cancer cell proliferation and vascularization. Identification of valuable biological processes and genes can be helpful for the understanding of the molecular mechanism of PDAC.
\end{abstract}

Correspondence to: Dr Guoquan Cao, Department of Radiology, The First Affiliated Hospital of Wenzhou Medical University, Wenzhou, Zhejiang, P.R. China

E-mail: 122257935@qq.com

Dr Mengtao Zhou, Department of Hepatobiliary Surgery, The First Affiliated Hospital of Wenzhou Medical University, Wenzhou, Zhejiang, P.R. China

E-mail:wz_zmt@163.com

Key words: pancreatic ductal adenocarcinoma, DEGs, miRNA, survival analysis

\section{Introduction}

Pancreatic cancer is the fourth most common cause of global cancer-related deaths (1) There were $\sim 44,000$ newly diagnosed cases of pancreatic cancer and more than 37,000 related deaths in 2012 in the US (2). In addition, the average 5-year survival rate is $\sim 7.7 \%$ based on the data from 2006 to 2012 (3). Pancreatic ductal adenocarcinoma (PDAC), the most common histological subtype, comprises $90 \%$ of all pancreatic cancer cases (4). PDAC always displays local invasion and distant metastasis during early stages leading to poor prognosis with an overall 5-year survival rate of only 5\% (5). World Health Organization (WHO) and Surveillance, Epidemiology and End Results (SEER) mortality data indicate that the occurrence of PDAC increases with age ( 71 years) (6). In addition, researchers have shown that various risk factors can contribute to the development of PDAC. In Italy, PDAC risk was found to be 4.3 -fold higher in heavy smokers ( $>20$ cigarettes/day) compared with never smokers (7). In addition, alcohol intake was found to be associated with PDAC mortality based on data from the Cancer Prevention Study II. The results show evidence that alcohol consumption promotes PDAC mortality (8).

To explore the molecular mechanism of PDAC, numerous studies have been carried out using advanced microarray or next-generation sequencing technology. Previous studies based on microarrays have identified several genes that play an important role in PDAC. GNAI2, G protein subunit $\alpha \mathrm{i} 2$, was found to be significantly upregulated in PDAC, and can mediate the functions of dopamine receptor D2 (DRD2) on cAMP signaling. Knockdown or inhibition of $D R D 2$ was found to reduce the proliferation of PDAC cells (9). Teodorczyk at al revealed that $C D 95$ is associated with stemness and epithelial-mesenchymal transition (EMT) in PDAC based on an in silico analysis of 36 RNA profiles (10). An in vitro experiment demonstrated that PDAC growth and metastasis can be significantly reduced by pharmacological inhibition of $C D 95$ activity, and $S c k$ is necessary for the $C D 95$ induction of cell cycle progression (10). In addition, expression levels of microRNAs and lncRNAs were also explored in PDAC, and several markers have been identified including miR-10b, miR-155, miR-106b (11) and lncRNA AFAPI-ASl (12). Recently, whole-exome sequencing of 109 micro-dissected PDAC samples identified multiple novel mutated genes in PDAC such as RBM10, KRAS, BRAF and high-frequency 
Table I. The identified 76 common DEGs in GSE32676 and GSE71989.

\begin{tabular}{|c|c|c|c|c|c|}
\hline \multirow[b]{2}{*}{ Gene } & \multicolumn{2}{|c|}{ Fold-change } & \multirow[b]{2}{*}{ Gene } & \multicolumn{2}{|c|}{ Fold-change } \\
\hline & GSE32676 & GSE71989 & & GSE32676 & GSE71989 \\
\hline CCL2 & -3.71 & 2.77 & LAMA3 & 3.75 & 2.80 \\
\hline TMC5 & 3.33 & 2.93 & $N R 4 A 2$ & -2.28 & 2.50 \\
\hline GJB2 & 4.54 & 2.58 & НОХВ3 & 2.08 & 2.09 \\
\hline DPCRI & 4.23 & 2.29 & EFNA5 & 2.12 & 2.16 \\
\hline MMP11 & 2.88 & 2.92 & $A N L N$ & 2.39 & 2.08 \\
\hline$C C L 8$ & -3.23 & 2.12 & CTSE & 5.12 & 3.49 \\
\hline$L C N 2$ & 4.59 & 2.18 & ANO1 & 2.83 & 2.80 \\
\hline ZWINT & 2.30 & 2.12 & MTUS2 & -2.32 & -2.39 \\
\hline$I F I 27$ & 2.10 & 3.37 & TSPAN1 & 4.41 & 2.18 \\
\hline MMP19 & -3.00 & 2.39 & C15orf48 & 3.77 & 2.57 \\
\hline$N Q O 1$ & 3.09 & 3.41 & $M M P 28$ & 3.06 & 2.26 \\
\hline SLC6A14 & 5.88 & 3.34 & C19orf33 & 5.67 & 2.99 \\
\hline ADAMTS12 & 2.69 & 2.41 & $L A M C 2$ & 2.25 & 2.37 \\
\hline$R R M 2$ & 2.40 & 2.38 & VILL & 2.98 & 2.10 \\
\hline SFTA2 & 4.14 & 2.27 & SERPINB5 & 5.62 & 2.29 \\
\hline PTGDS & -2.79 & 2.17 & CAMK2N1 & 2.08 & 2.47 \\
\hline$L A M B 3$ & 4.87 & 2.48 & ST6GALNAC1 & 3.75 & 2.61 \\
\hline$G P R C 5 A$ & 3.14 & 3.46 & ETV1 & 2.18 & 2.38 \\
\hline PHLDA2 & 3.04 & 3.40 & $D C B L D 1$ & 2.26 & 2.40 \\
\hline PPPIRIA & -2.42 & -2.03 & CST1 & 4.90 & 2.25 \\
\hline OAS1 & 2.51 & 2.10 & GCNT3 & 3.93 & 2.38 \\
\hline$E C T 2$ & 2.40 & 3.04 & SOCS3 & -2.43 & 3.25 \\
\hline SDR16C5 & 4.78 & 3.21 & MUC4 & 4.30 & 2.44 \\
\hline LOC100505984 & 4.96 & 3.34 & $S D C 1$ & 2.68 & 2.36 \\
\hline$M A L L$ & 2.13 & 2.34 & EPPK1 & 3.54 & 2.31 \\
\hline THBSI & -3.11 & 2.60 & $A G R 2$ & 3.96 & 2.45 \\
\hline CEACAM1 & 3.47 & 2.08 & $A G R 3$ & 3.76 & 3.11 \\
\hline$C L D N 23$ & 3.21 & 2.21 & $S F N$ & 2.64 & 3.75 \\
\hline$K L F 5$ & 3.09 & 2.09 & $C D K 1$ & 2.60 & 2.21 \\
\hline CEACAM5 & 6.81 & 3.96 & $O G N$ & -3.20 & 2.42 \\
\hline$I T G A 2$ & 3.33 & 2.65 & $A O C 1$ & 3.76 & 2.44 \\
\hline СЕАСАM & 5.85 & 4.22 & $E M P 1$ & -2.63 & 3.38 \\
\hline KRT17 & 4.01 & 3.00 & S100P & 6.75 & 4.78 \\
\hline S100A6 & 3.11 & 2.92 & $A H N A K 2$ & 3.76 & 2.55 \\
\hline$F O S B$ & -3.97 & 3.40 & $M S L N$ & 6.44 & 3.48 \\
\hline$T F F 1$ & 5.06 & 2.92 & CAPN8 & 5.28 & 2.99 \\
\hline TOP2A & 2.76 & 3.21 & KRT19 & 6.22 & 3.93 \\
\hline$L Y 6 E$ & 2.16 & 2.37 & $\mathrm{CH} 25 \mathrm{H}$ & -2.42 & 2.06 \\
\hline
\end{tabular}

DEGs, differentially expressed genes.

alterations in Wnt signaling, chromatin remodelling and cell cycle pathways (13).

Research has been carried out to explore the molecular mechanisms of PDAC based on microarray expression profiles or next-generation sequencing. However, studies with the integration of mRNA and miRNA expression profiles have not been widely applied in PDAC. In recent years, more and more microarray expression datasets have been submitted to the Gene Expression Omnibus (GEO) database, and re-analysis of the deposited datasets with various bioinformatics algorithms can be helpful (14). In the present study, we firstly identified the common differentially expressed genes (DEGs) in PDAC based on 2 mRNA expression profiles from 2 independent laboratories. Then functional annotation of the common DEGs based on Gene Ontology (GO) and Kyoto Encyclopedia of Genes and Genomes (KEGG) pathway databases was carried out. Furthermore, an interaction network between the identified DEGs and documented miRNAs was constructed. Finally, the identified DEGs were virtually validated using SurvExpress online database. 

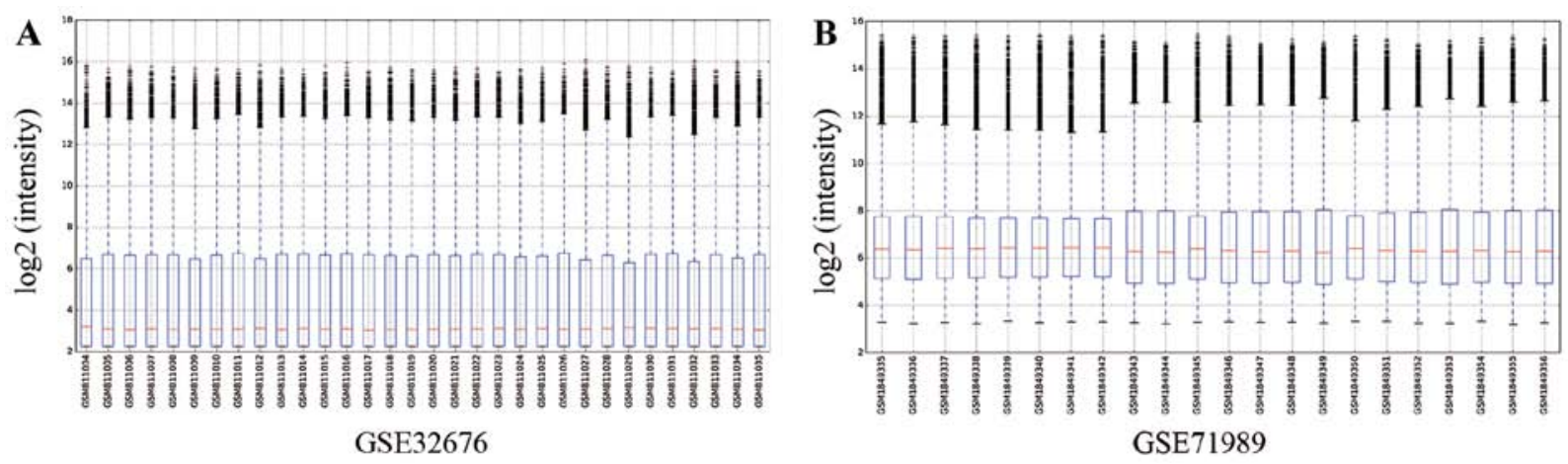

Figure 1. Boxplots for the gene expression of each sample in (A) GSE32676 and (B) GSE71989.

\section{Materials and methods}

Acquisition of $m R N A$ and miRNA expression profiles. In the present study, publicly available datasets from Expression Omnibus Database (GEO) (http://www.ncbi.nlm.nih.gov/geo/) were used. Firstly, we carefully searched the GEO database, and downloaded 2 mRNA expression profiles. GSE71989 submitted by Thomas Schmittgen in 2015 (https://www.ncbi. nlm.nih.gov/geo/query/acc.cgi?acc=GSE71989) consists of 14 PDAC and 8 normal pancreas tissues. The other dataset GSE32676 submitted by Tran in 2011 (15) consists of 25 PDAC and 7 normal pancreas tissues. Based on the instruction from manufacturer, RNAs were extracted and hybridized to Affymetrix Human Genome U133 Plus 2.0 array. Detailed sample information and experiment designs were documented in the previous studies.

Differentially expressed gene screening. Data analysis was carried out using in-house $\mathrm{R}$ script and publicly available annotation database. In brief, mRNA expression values were firstly subject to background correction, normalization and $\log _{2}$ transformation using GeneChip Robust Multi-array Analysis (GC-RMA) algorithm (16). Furthermore, uninformative control probe sets were filtered out. In addition, the average expression value was calculated for the genes with multiple probes. Finally, DEGs were screened using Linear Models for Microarray Data (Limma) package (17) within the bioconductor. The criteria were set to adjust $\mathrm{p}$-value $\leq 0.05$ and $\mid \log _{2}$ fold-change $(F C) \mid \geq 2$. In addition, the common DEGs between the 2 datasets were identified based on a Venn diagram. The common DEGs were used to construct a heat map using Heatmap.2 method within ggplot package (18).

GO and KEGG pathway annotation. The functions of the identified DEGs were further annotated using GO and KEGG pathway databases using the online tools of Database for Annotation, Visualization and Integrated Discovery (DAVID) (19). The GO term consist of biological process (BP), cellular component (CC) and molecular function (MF). The criterion was set to $\mathrm{p}<0.05$.

mRNA-miRNA interaction network. Numerous studies show that miRNAs play an important role in the regulation of carcinogenesis, malignant transformation and metastatic processes by preventing mRNA expression or via other processes (20).
Cote et al (11) showed that 5 miRNAs, miR-10b-5p, miR-155-5p, miR-106b-5p, miR-30c-5p and miR-212-3p, have excellent performance to distinguish PDAC from normal samples. In addition, the sensitivity and specificity were 96 and $100 \%$, respectively, in the training and validation cohorts. In the present study, we constructed the mRNA-miRNA interaction network based on the common DEGs and the 5 miRNAs. In brief, target genes of the 5 miRNAs were predicted based on the microCosm, mirTarbase and TargetScan databases. Then, the intersection between the common DEGs and the target genes were selected. Finally, the interaction network was constructed using CyTargetLinker (21) plugin in Cytoscape (22).

Virtual validation of the common DEGs. Clinical outcomes of the DEGs are critical for the diagnosis or treatment of PDAC. In the present study, virtual validation of the DEGs was carried out using SurvExpress online tool (23). This tool is based on a cancer-wide gene expression database with clinical outcomes. Four datasets were used for the virtual validation including GSE21501, GSE28735, TCGA PDAC and ICGC PDAC. Detailed information for the datasets can be found in previous studies. Parameter setting were carefully selected according to the developer's instructions.

\section{Results}

$D E G s$ in PDAC. After background correction and normalization, the gene expression median values for different samples in the 2 datasets were almost at the same level (Fig. 1). Then, the datasets were subjected to DEG analysis. Results showed that a total of 364 and 816 DEGs were screened out for GSE32676 and GSE71989, respectively. For GSE32676, 292 genes (80.2\%) were upregulated and 72 genes $(19.8 \%)$ were downregulated. For GSE71989, 666 genes (81.6\%) were upregulated and 150 genes $(18.4 \%)$ were downregulated. Among those DEGs, 76 genes were differentially expressed in both GSE32676 and GSE71989 (Fig. 2). Fold-changes for the common DEGs are listed in Table I.

Furthermore, all samples from the 2 datasets were subjected to hierarchical clustering analysis based on the common DEGs. As shown in Fig. 3, tumor (red) and normal (blue) samples were abe to be clearly classified into different subgroups. Due to tumor heterogeneity and expression value variation, a few normal and tumor samples were erroneously classified. 


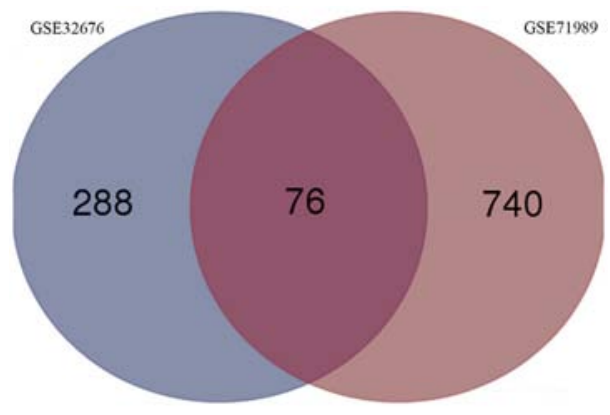

Figure 2. Venn diagram of the differentially expressed genes (DEGs) in GSE32676 and GSE71989.
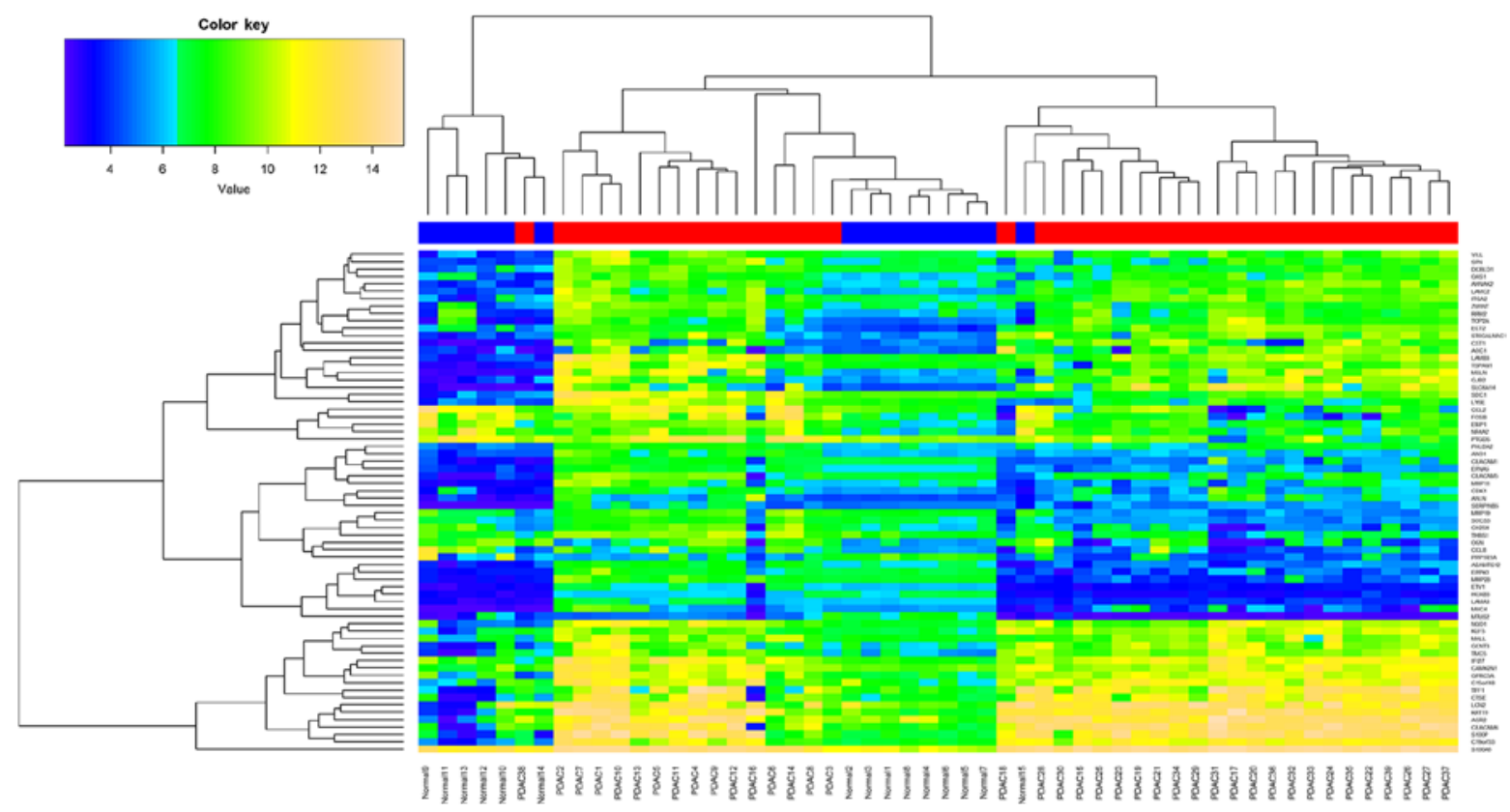

Figure 3. Heat map showing the differential expression pattern of 76 genes out of 1,104 differentially expressed genes (DEGs) in all samples. The x-axis represents samples and the bar on the top indicates the tumor samples (red) and the normal samples (blue). The y-axis represents the 76 genes.

Table II. KEGG pathway enrichment result for the common DEGs.

\begin{tabular}{lll}
\hline Term & \multicolumn{1}{c}{ Genes } & P-value \\
\hline hsa04512:ECM-receptor interaction & LAMB3, SDC1, LAMA3, ITGA2, LAMC2, THBS1 & $1.39 \mathrm{E}-05$ \\
hsa04115:p53 signaling pathway & CDK1, SERPINB5, RRM2, SFN, THBS1 & $1.21 \mathrm{E}-04$ \\
hsa05222:Small cell lung cancer & LAMB3, LAMA3, ITGA2, LAMC2 & 0.0040471 \\
hsa04510:Focal adhesion & LAMB3, LAMA3, ITGA2, LAMC2, THBS1 & 0.0069739
\end{tabular}

KEGG, Kyoto Encyclopedia of Genes and Genomes; DEGs, differentially expressed genes.

GO and KEGG pathway annotation. In order to explore the biological functions of the common DEGs, functional annotation of the common DEGs was carried out using DAVID online tool based on GO and KEGG pathway databases. Results showed that the common DEGs can be significantly enriched into 4 KEGG pathways (Table II). Six genes participated in the ECM-receptor interaction pathway $(\mathrm{p}=0.000013), 5$ genes were involved in the $\mathrm{p} 53$ signaling pathway ( $\mathrm{p}=0.00012), 4$ genes mapped to small cell lung cancer $(\mathrm{p}=0.0040)$ and 5 genes play a role in the pathway of focal adhesion ( $\mathrm{p}=0.0069)$. In addition, the common DEGs were mainly related to the biological process of epidermis development $(\mathrm{p}=0.0001)$, ectoderm development $(\mathrm{p}=0.0002)$ and cell adhesion ( $\mathrm{p}=0.0008)$ (Table III). In addition, the top 5 cellular components include extracellular matrix $(\mathrm{p}=0.0)$, extracellular region $(\mathrm{p}=0.0)$, extracellular region part $(\mathrm{p}=0.0)$, proteinaceous extracellular matrix $(\mathrm{p}=0.0002)$ and anchored to membrane ( $\mathrm{p}=0.023)$ (Table III). In addition, 5 signifi- 
Table III. Top 5 GO terms for the common DEGs.

\begin{tabular}{|c|c|c|c|}
\hline ID & GO term & P-values & Genes \\
\hline \multicolumn{4}{|l|}{ BP } \\
\hline GO:0008544 & Epidermis development & 0.0001 & LAMB3, LAMA3, KRT17, AHNAK2, LAMC2, SFN, EMP1 \\
\hline GO:0007398 & Ectoderm development & 0.0002 & LAMB3, LAMA3, KRT17, AHNAK2, LAMC2, SFN, EMP1 \\
\hline GO:0007155 & Cell adhesion & 0.0008 & $\begin{array}{l}\text { DCBLD1, LAMB3, LAMA3, CCL2, MSLN, ITGA2, LAMC2, } \\
\text { THBS1, CEACAM1, CLDN23, MUC4 }\end{array}$ \\
\hline GO:0022610 & Biological adhesion & 0.0008 & $\begin{array}{l}\text { DCBLD1, LAMB3, LAMA3, CCL2, MSLN, ITGA2, LAMC2, } \\
\text { THBS1, CEACAM1, CLDN23, MUC4 }\end{array}$ \\
\hline GO:0048545 & $\begin{array}{l}\text { Response to steroid } \\
\text { hormone stimulus }\end{array}$ & 0.0015 & KRT19, SDC1, CCL2, SOCS3,TFF1, THBS1 \\
\hline \multicolumn{4}{|l|}{$\mathrm{CC}$} \\
\hline GO:0031012 & Extracellular matrix & 0.0000 & $\begin{array}{l}\text { OGN, LAMB3, LAMA3, MMP19, LAMC2, MMP28, } \\
\text { ADAMTS12,THBS1, MMP11, MUC4 }\end{array}$ \\
\hline GO:0005576 & Extracellular region & 0.0000 & $\begin{array}{l}\text { CCL2, MMP19, CCL8, CST1, MMP28, SFN, MUC4, MMP11, } \\
L C N 2, \text { OGN, LAMB3, LAMA3, PTGDS, SERPINB5, MSLN, } \\
\text { SFTA2, LAMC2, EFNA5, ADAMTS12, TFF1, AGR3, } \\
\text { THBS1, AGR2, CEACAM1 }\end{array}$ \\
\hline GO:0044421 & Extracellular region part & 0.0000 & $\begin{array}{l}\text { CCL2, MMP19, CCL8, MMP28, SFN, MMP11, MUC4, OGN } \\
\text { LAMB3, LAMA3, SERPINB5, LAMC2, EFNA5, TFF1, } \\
\text { ADAMTS12, THBS1 }\end{array}$ \\
\hline GO:0005578 & $\begin{array}{l}\text { Proteinaceous extracellular } \\
\text { matrix }\end{array}$ & 0.0002 & $\begin{array}{l}\text { OGN, LAMB3, LAMA3, MMP19, LAMC2, MMP28, } \\
\text { ADAMTS12, MMP11, MUC4 }\end{array}$ \\
\hline GO:0031225 & Anchored to membrane & 0.0231 & LY6E, MSLN, CEACAM6, CEACAM5, EFNA5 \\
\hline \multicolumn{4}{|l|}{ MF } \\
\hline GO:0005198 & Structural molecule activity & 0.0035 & $\begin{array}{l}\text { KRT19, LAMB3, LAMA3, EPPK1, KRT17, THBS1, VILL, } \\
\text { CLDN23, MUC4 }\end{array}$ \\
\hline GO:0004857 & Enzyme inhibitor activity & 0.0044 & SERPINB5, SOCS3, PPP1R1A, CST1, SFN, CAMK2N1 \\
\hline GO:0008201 & Heparin binding & 0.0081 & CCL2, CCL8, LAMC2, THBS1 \\
\hline GO:0004222 & Metalloendopeptidase activity & 0.0083 & MMP19, MMP28, ADAMTS12, MMP11 \\
\hline GO:0004860 & Protein kinase inhibitor activity & 0.0091 & $S O C S 3, S F N, C A M K 2 N 1$ \\
\hline
\end{tabular}

GO, Gene Ontology; DEGs, differentially expressed genes; BP, biological process; CC, cellular component; MF, molecular function.

Table IV. Virtual validation results of the common DEGs using 4 PDAC datasets.

\begin{tabular}{|c|c|c|c|c|c|c|}
\hline \multirow[b]{2}{*}{ Dataset } & \multirow[b]{2}{*}{ Samples } & \multirow{2}{*}{$\begin{array}{l}\text { Genes } \\
\text { found }\end{array}$} & \multirow{2}{*}{$\begin{array}{c}\text { Risk groups } \\
\text { p-value }\end{array}$} & \multirow[b]{2}{*}{ CI } & \multicolumn{2}{|r|}{ DEGs between risk groups } \\
\hline & & & & & No. & DEGs \\
\hline TCGA & 176 & 75 & $9.90 \mathrm{E}-15$ & 79.6 & 49 & TMC5, GJB2, DPCR1, MMP11, ZWINT... \\
\hline ICGC & 189 & 75 & $0.00 \mathrm{E}+00$ & 82.1 & 17 & 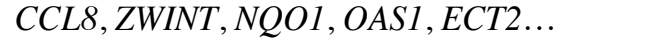 \\
\hline GSE21501 & 132 & 75 & $6.00 \mathrm{E}-13$ & 95.4 & 11 & SLC6A14, CEACAM5, FOSB, LAMA3, ANO1.. \\
\hline GSE28735 & 90 & 73 & $1.10 \mathrm{E}-06$ & 99.9 & 4 & RRM2, PTGDS, ITGA2, C15orf 48 \\
\hline
\end{tabular}

DEGs, differentially expressed genes; PDAC, pancreatic ductal adenocarcinoma; CI, concordance index.

cant molecular function were enriched for the common DEGs including structural molecule $(\mathrm{p}=0.0035)$, enzyme inhibitor $(p=0.0044)$, heparin binding $(p=0.0081)$, metalloendopeptidase $(\mathrm{p}=0.0083)$ and protein kinase inhibitor activities $(\mathrm{p}=0.0091)$ (Table III).
mRNA-miRNA network construction. To explore mRNA and miRNA regulation mechanism, the mRNA-miRNA pairings were constructed. The results indicated that the 5 miRNAs were able to target 3,760,827 and 3,671 genes in the MicroCosm, mirTarbase and TargetScan database, 


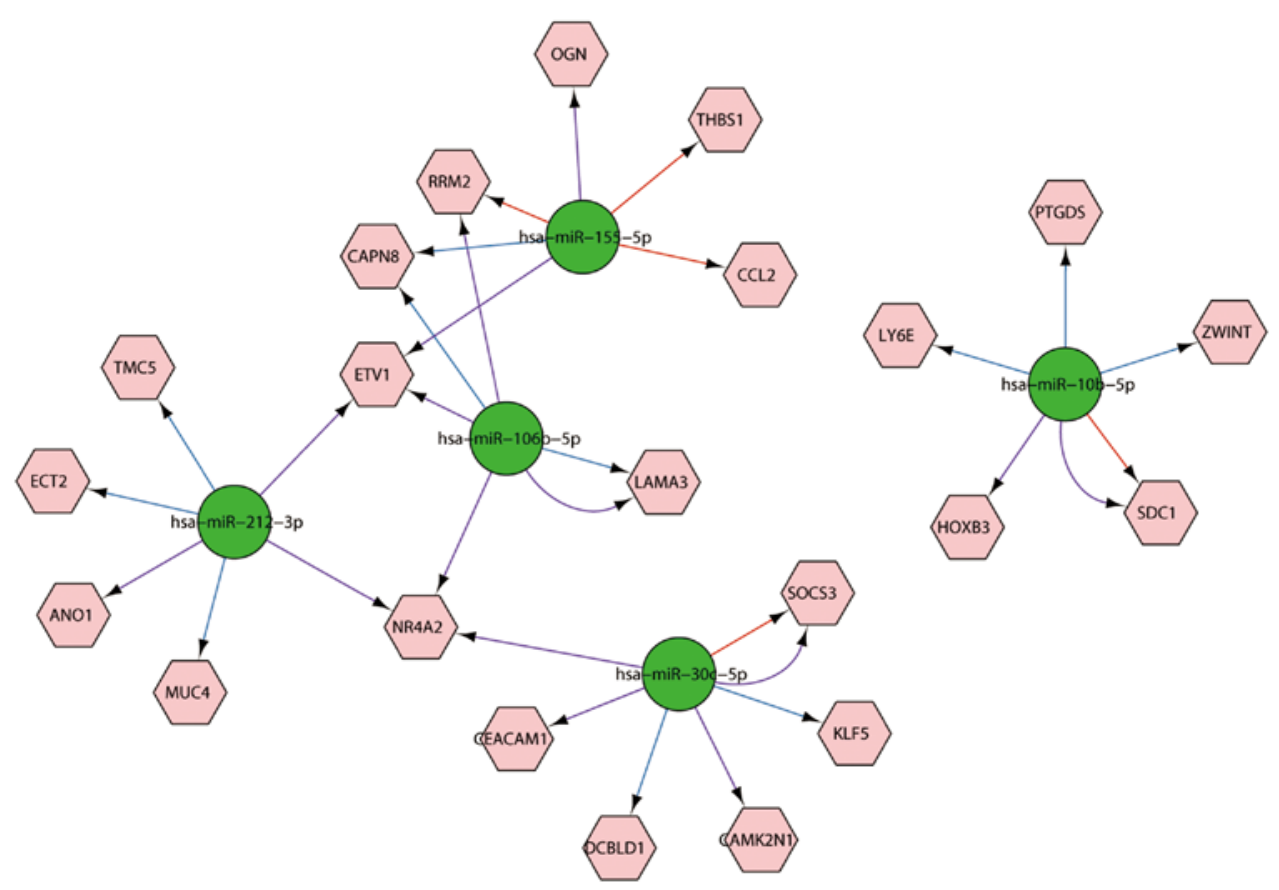

Figure 4. Interaction network between the common differentially expressed genes (DEGs) and 5 reported miRNAs. Pink hexagon represent DEGs and green circle represent miRNAs.
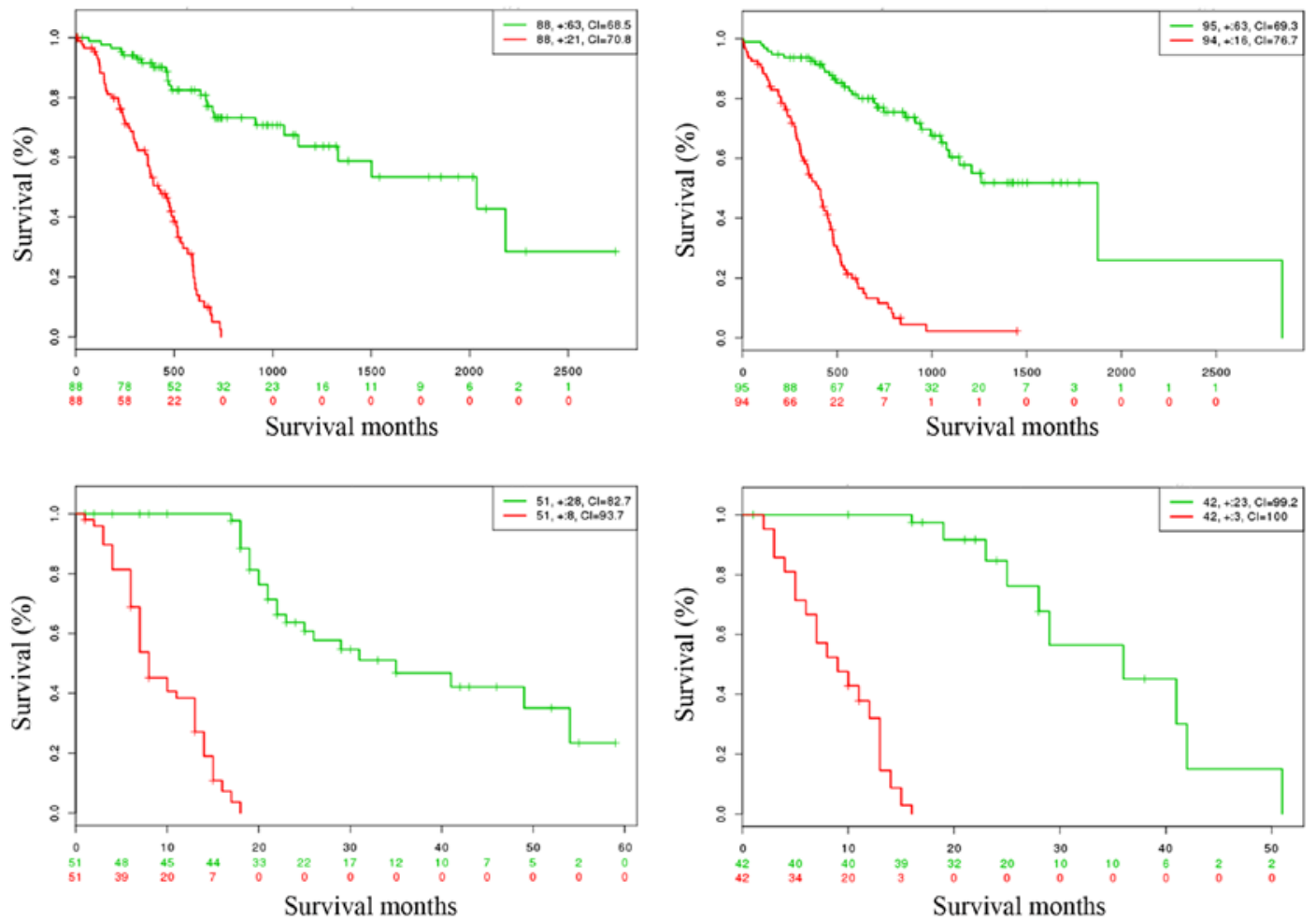

Figure 5. Kaplan-Meier curves of 4 bladder cancer datasets in SurvExpress database. Censored samples are marked with '+'. The x-axis represents time to event and y-axis represents percentage. The number of samples, censored number and concordance index (CI) are shown in the top-right insets. High- and low-risk groups are labeled with red and green curves, respectively.

respectively. Among those, 22 genes were also identified in the common DEGs. Then, the interaction network between the 22 genes and 5 miRNAs were constructed (Fig. 4). Based on the figure, we found that hsa-miR-212-3p can regulate 6 genes, hsa-miR-106p-5p can regulate 5 genes, hsa-miR-155-5p can regulate 5 genes, hsa-miR-10b-5p can regulate 5 genes and hsa-miR-30c-5p can regulate 6 genes.

Virtual validation. Biomarker validation is critical in the study of cancer molecular mechanism research. The prognostic 
performance of the common DEGs were validated using the SurvExpress online tool which provides survival analysis and risk assessment. Results are shown in Fig. 5 and are summarized in Table IV. The figure indicated that low- and high-risk PDAC groups can be significantly differentiated in the 4 datasets, and the p-values were 9.9e-15, 0.0, 6.0e-13 and $1.1 \mathrm{e}-06$, respectively. In addition, the higher concordance index (CI) demonstrated that better prediction results can be achieved based on those DEGs (Table IV).

\section{Discussion}

With the development of microarray and next generation sequencing technologies, understanding of the molecular mechanism of pancreatic ductal adenocarcinoma (PDAC) has been significantly advanced. In the present study, we aimed to unveil the complex mechanism of PDAC by integrating different types of omics data. A total of 76 genes were simultaneously differentially expressed in 39 tumor tissues. Notably, a heat map showed that tumor and normal samples were able to be clearly distinguished based on the DEGs. The erroneous assignment of 2 samples was probably due to tumor heterogeneity.

KEGG pathway enrichment revealed that the DEGs were involved in the ECM-receptor interaction pathway. Studies have shown that PDAC is characterized by prominent desmoplasia (24). Extracellular matrix (ECM) proteins, ECM metabolizing enzymes and growth factors are main components of desmoplasia, and the components can promote the growth of cancer cells (25). In addition, ECM proteins and desmoplastic secreted growth factors can activate intracellular signals including reactive oxygen species that prevent the death of PDAC cancer cells (25). In addition, several ECM components such as collagens I/III/IV, decorin and versican may be of clinical prognostic significance in PDAC (26). Another significantly enriched pathway is focal adhesion. Researches have demonstrated that interaction between integrin and focal adhesion kinase can regulate cancer cell adhesion and invasion $(27,28)$. Sawai et al showed that phosphorylation of focal adhesion kinase is involved in the aggressive capability of PDAC via the Ras/ERK signaling pathway (29). In addition, MUC16, a heavily glycosylated type-I transmembrane mucin, can facilitate PDAC growth and metastasis via focal adhesion signaling (30).

The mRNA and miRNA interaction network analysis further unveiled the complex mechanism of PDAC. The interaction network showed that hsa-miR-212-3p can regulate the DEG MUC4. This gene encodes highly glycosylated integral membrane glycoprotein in the cell surface (31). In addition, immunohistochemical analyses based on 135 PDAC tissues demonstrated that MUC4 was significantly highly expressed in patients with poor prognosis $(\mathrm{p}=0.0043)(32)$. In addition, hypomethylation of the MUC4 promoter probably participated in the carcinogenesis and malignant development of PDAC based on DNA methylation-specific PCR analysis of 116 microdissected foci (33). Jonckheere et al showed that MUC4 can interact with the ErbB2 oncogenic receptor via EGF domains, and inhibition of MUC4 expression can affect the downstream JNK pathway (34).

Another important gene is $R R M 2$ which encodes one of 2 non-identical subunits for ribonucleotide reductase.
Ribonucleotide reductase has been demonstrated to be a determinant of gemcitabine chemoresistance in human cancers (35), and the level of RRM2 can regulate enzyme activity (36). Duxbury et al showed that high expression of $R R M 2$ is related to gemcitabine chemoresistance in PDAC (37). The suppression of RRM2 by siRNA significantly inhibited tumor growth, metastasis and increased tumor apoptosis (37). Research has also shown that $N F-\kappa B$ is the key mediator by which $R R M 2$ induces invasiveness in PDAC (38). RRM2 and its downstream intermediaries can become potential drug targets (38). The mRNA-miRNA interaction network demonstrated that hsamiR-106p-5p and hsa-miR-155-5p can regulate $R R M 2$.

In addition, the CCL2 gene, which is also refered to as monocyte chemoattractant protein 1 and is a small cytokine (39), has been widely reported to be related to PDAC progression. In PDAC, the expression of CCL2 was found to be significantly elevated (40). In addition, Kalbasi et al demonstrated that highly expressed $C C L 2$ can recruit $\mathrm{Ly} 6 \mathrm{C}^{+} \mathrm{CCR} 2^{+}$inflammatory monocytes or macrophages to the regions surrounding the tumor and promote tumor proliferation and vascularization (41). In addition, PDAC cancer cells can construct an immunosuppressive tumor microenvironment by recruiting regulatory $T$ cells with the high-expression of CCL5 (42). The mRNA-miRNA interaction network showed that hsa-miR-155-5p can regulate CCL2.

Apart from the above-mentioned 3 critical genes in PDAC, some rarely reported or novel genes such as ECT2, SDC1, $S O C S 3, T M C 5$ and NR4A2, also play a role in the development of PDAC. The ECT2 gene can promote Rho activity during cytokinesis, and RT-PCR results showed that it was highly expressed in PDAC (43). Statistical analysis showed that epithelial expression of SDC1 was positively correlated with survival time in PDAC patients $(\mathrm{p}=0.029)(44)$. Lesina et al showed that homozygous deletion of Socs 3 can aberrantly activate Stat 3 and promote PDAC development (45).

In summary, the development and progression of PDAC were probably induced via various processes. Firstly, activation of MUC4 induces nuclear translocation of $\beta$-catenin and promotes the process of angiogenesis that can provide necessary nutrition or oxygen for cancer cells. Then, $R R M 2$ can induce the invasiveness of PDAC via $N F-\kappa B$. Finally, the formation of an immunosuppressive tumor microenvironment by recruiting regulatory $\mathrm{T}$ cells with high expression of CCL2 further promotes cancer cell proliferation and vascularization.

\section{Acknowledgements}

The present study was funded by the Natural Science Foundation of Zhejiang Province (nos. LY15H030016 and LY15H160056) and Wenzhou Science \& Technology Bureau (no. Y20140696).

\section{References}

1. Hariharan D, Saied A and Kocher HM: Analysis of mortality rates for pancreatic cancer across the world. HPB 10: 58-62, 2008.

2. American Cancer Society: Cancer Facts \& Figures 2012. American Cancer Society, Atlanta, GA, USA, 2012.

3. Stat Fact Sheets SEER: Pancreas Cancer. National Cancer Institute, 2013.

4. Ishiwata T: Pancreatic ductal adenocarcinoma: Basic and clinical challenges for better prognosis. J Carcinog Mutagen S9: 2157-2518, 2013. http://dx.doi.org/4172/2157-2518.S9-005. 
5. Siegel R, Ward E, Brawley O and Jemal A: Cancer statistics, 2011: The impact of eliminating socioeconomic and racial disparities on premature cancer deaths. CA Cancer J Clin 61: 212-236, 2011.

6. Partensky C: Toward a better understanding of pancreatic ductal adenocarcinoma: Glimmers of hope? Pancreas 42: 729-739, 2013

7. Talamini R, Polesel J, Gallus S, Dal Maso L, Zucchetto A, Negri E, Bosetti C, Lucenteforte E, Boz G, Franceschi S, et al: Tobacco smoking, alcohol consumption and pancreatic cancer risk: A case-control study in Italy. Eur J Cancer 46: 370-376, 2010.

8. Gapstur SM, Jacobs EJ, Deka A, McCullough ML, Patel AV and Thun MJ: Association of alcohol intake with pancreatic cancer mortality in never smokers. Arch Intern Med 171: 444-451, 2011.

9. Jandaghi P, Najafabadi HS, Bauer AS, Papadakis AI, Fassan M, Hall A, Monast A, von Knebel Doeberitz M, Neoptolemos JP, Costello E, et al: Expression of DRD2 is increased in human pancreatic ductal adenocarcinoma and inhibitors slow tumor growth in mice. Gastroenterology 151: 1218-1231, 2016.

10. Teodorczyk M, Kleber S, Wollny D, Sefrin JP, Aykut B, Mateos A, Herhaus P, Sancho-Martinez I, Hill O, Gieffers C, et al: CD95 promotes metastatic spread via Sck in pancreatic ductal adenocarcinoma. Cell Death Differ 22: 1192-1202, 2015.

11. Cote GA, Gore AJ, McElyea SD, Heathers LE, Xu H, Sherman S and Korc M: A pilot study to develop a diagnostic test for pancreatic ductal adenocarcinoma based on differential expression of select miRNA in plasma and bile. Am J Gastroenterol 109: 1942-1952, 2014.

12. Ye Y, Chen J, Zhou Y, Fu Z, Zhou Q, Wang Y, Gao W, Zheng S, Zhao X, Chen T, et al: High expression of AFAPI-AS1 is associated with poor survival and short-term recurrence in pancreatic ductal adenocarcinoma. J Transl Med 13: 137, 2015.

13. Witkiewicz AK, McMillan EA, Balaji U, Baek G, Lin WC Mansour J, Mollaee M, Wagner KU, Koduru P, Yopp A, et al: Whole-exome sequencing of pancreatic cancer defines genetic diversity and therapeutic targets. Nat Commun 6: 6744, 2015

14. Berger B, Peng J and Singh M: Computational solutions for omics data. Nat Rev Genet 14: 333-346, 2013.

15. Donahue TR, Tran LM, Hill R, Li Y, Kovochich A, Calvopina JH, Patel SG, Wu N, Hindoyan A, Farrell JJ, et al: Integrative survival-based molecular profiling of human pancreatic cancer. Clin Cancer Res 18: 1352-1363, 2012.

16. Gentleman RC, Carey VJ, Bates DM, Bolstad B, Dettling M, Dudoit S, Ellis B, Gautier L, Ge Y, Gentry J, et al: Bioconductor: Open software development for computational biology and bioinformatics. Genome Biol 5: R80, 2004.

17. Kerr MK: Linear models for microarray data analysis: Hidden similarities and differences. J Comput Biol 10: 891-901, 2003.

18. Hadley W: ggplot2: Elegant Graphics for Data Analysis. Springer, Switzerland, 2016

19. Dennis G Jr, Sherman BT, Hosack DA, Yang J, Gao W, Lane HC and Lempicki RA: DAVID: Database for Annotation, Visualization, and Integrated Discovery. Genome Biol 4: 3, 2003.

20. Acunzo M and Croce CM: MicroRNA in cancer and cachexia - A mini-review. J Infect Dis 212 (Suppl 1): S74-S77, 2015.

21. Kutmon M, Kelder T, Mandaviya P, Evelo CT and Coort SL: CyTargetLinker: A cytoscape app to integrate regulatory interactions in network analysis. PLoS One 8: e82160, 2013.

22. Shannon P, Markiel A, Ozier O, Baliga NS, Wang JT, Ramage D, Amin N, Schwikowski B and Ideker T: Cytoscape: A software environment for integrated models of biomolecular interaction networks. Genome Res 13: 2498-2504, 2003.

23. Aguirre-Gamboa R, Gomez-Rueda H, Martínez-Ledesma E, Martínez-Torteya A, Chacolla-Huaringa R, RodriguezBarrientos A, Tamez-Peña JG and Treviño V: SurvExpress: An online biomarker validation tool and database for cancer gene expression data using survival analysis. PLoS One 8: e74250, 2013

24. Mollenhauer J, Roether I and Kern HF: Distribution of extracellular matrix proteins in pancreatic ductal adenocarcinoma and its influence on tumor cell proliferation in vitro. Pancreas 2: 14-24, 1987.

25. Pandol S, Edderkaoui M, Gukovsky I, Lugea A and Gukovskaya A: Desmoplasia of pancreatic ductal adenocarcinoma. Clin Gastroenterol Hepatol 7 (Suppl 11): S44-47, 2009.

26. Mahadevan D and Von Hoff DD: Tumor-stroma interactions in pancreatic ductal adenocarcinoma. Mol Cancer Ther 6: 1186-1197, 2007.

27. Crowe DL and Ohannessian A: Recruitment of focal adhesion kinase and paxillin to betal integrin promotes cancer cell migration via mitogen activated protein kinase activation. BMC Cancer 4: 18, 2004.
28. Schmitz KJ, Grabellus F, Callies R, Otterbach F, Wohlschlaeger J, Levkau B, Kimmig R, Schmid KW and Baba HA: High expression of focal adhesion kinase $\left(\mathrm{p} 125^{\mathrm{FAK}}\right)$ in node-negative breast cancer is related to overexpression of HER-2/neu and activated Akt kinase but does not predict outcome. Breast Cancer Res 7: R194-R203, 2005.

29. Sawai H, Okada Y, Funahashi H, Matsuo Y, Takahashi H, Takeyama $\mathrm{H}$ and Manabe T: Activation of focal adhesion kinase enhances the adhesion and invasion of pancreatic cancer cells via extracellular signal-regulated kinase-1/2 signaling pathway activation. Mol Cancer 4: 37, 2005.

30. Muniyan S, Haridas D, Chugh S, Rachagani S, Lakshmanan I, Gupta S, Seshacharyulu P, Smith LM, Ponnusamy MP and Batra SK: MUC16 contributes to the metastasis of pancreatic ductal adenocarcinoma through focal adhesion mediated signaling mechanism. Genes Cancer 7: 110-124, 2016.

31. Mall AS: Analysis of mucins: Role in laboratory diagnosis. J Clin Pathol 61: 1018-1024, 2008

32. Saitou M, Goto M, Horinouchi M, Tamada S, Nagata K, Hamada T, Osako M, Takao S, Batra SK, Aikou T, et al: MUC4 expression is a novel prognostic factor in patients with invasive ductal carcinoma of the pancreas. J Clin Pathol 58: 845-852, 2005.

33. Zhu Y, Zhang JJ, Zhu R, Zhu Y, Liang WB, Gao WT, Yu JB, $\mathrm{Xu} \mathrm{ZK}$ and Miao Y: The increase in the expression and hypomethylation of MUC4 gene with the progression of pancreatic ductal adenocarcinoma. Med Oncol 28 (Suppl 1): S175-S184, 2011.

34. Jonckheere N, Skrypek N, Merlin J, Dessein AF, Dumont P, Leteurtre E, Harris A, Desseyn JL, Susini C, Frénois F, et al: The mucin MUC4 and its membrane partner ErbB2 regulate biological properties of human CAPAN-2 pancreatic cancer cells via different signalling pathways. PLoS One 7: e32232, 2012.

35. Goan YG, Zhou B, Hu E, Mi S and Yen Y: Overexpression of ribonucleotide reductase as a mechanism of resistance to 2,2-difluorodeoxycytidine in the human KB cancer cell line. Cancer Res 59: 4204-4207, 1999.

36. Eriksson S and Martin DW Jr: Ribonucleotide reductase in cultured mouse lymphoma cells. Cell cycle-dependent variation in the activity of subunit protein M2. J Biol Chem 256: 9436-9440, 1981.

37. Duxbury MS, Ito H, Zinner MJ, Ashley SW and Whang EE: RNA interference targeting the M2 subunit of ribonucleotide reductase enhances pancreatic adenocarcinoma chemosensitivity to gemcitabine. Oncogene 23: 1539-1548, 2004.

38. Duxbury MS and Whang EE: RRM2 induces NF-kappaBdependent MMP-9 activation and enhances cellular invasiveness. Biochem Biophys Res Commun 354: 190-196, 2007.

39. Carr MW, Roth SJ, Luther E, Rose SS and Springer TA: Monocyte chemoattractant protein 1 acts as a T-lymphocyte chemoattractant. Proc Natl Acad Sci USA 91: 3652-3656, 1994.

40. Monti P, Leone BE, Marchesi F, Balzano G, Zerbi A, Scaltrini F, Pasquali C, Calori G, Pessi F, Sperti C, et al: The CC chemokine MCP-1/CCL2 in pancreatic cancer progression: Regulation of expression and potential mechanisms of antimalignant activity. Cancer Res 63: 7451-7461, 2003.

41. Kalbasi A, Komar CA, Tooker GM, Liu M, Lee JW, Gladney WL, Ben-Josef E and Beatty GL: Tumor-derived CCL2 mediates resistance to radiotherapy in pancreatic ductal adenocarcinoma. Clin Cancer Res 23: 137-148, 2017.

42. Tan MC, Goedegebuure PS, Belt BA, Flaherty B, Sankpal N, Gillanders WE, Eberlein TJ, Hsieh CS and Linehan DC: Disruption of CCR5-dependent homing of regulatory T cells inhibits tumor growth in a murine model of pancreatic cancer. $\mathrm{J}$ Immunol 182: 1746-1755, 2009.

43. Zhang ML, Lu S, Zhou L and Zheng SS: Correlation between ECT2 gene expression and methylation change of ECT2 promoter region in pancreatic cancer. Hepatobiliary Pancreat Dis Int 7: 533-538, 2008

44. Hrabar D, Aralica G, Gomercic M, Ljubicic N, Kruslin B and Tomas D: Epithelial and stromal expression of syndecan-2 in pancreatic carcinoma. Anticancer Res 30: 2749-2753, 2010.

45. Lesina M, Kurkowski MU, Ludes K, Rose-John S, Treiber M, Klöppel G, Yoshimura A, Reindl W, Sipos B, Akira S, et al: Stat $3 /$ Socs 3 activation by IL-6 transsignaling promotes progression of pancreatic intraepithelial neoplasia and development of pancreatic cancer. Cancer Cell 19: 456-469, 2011. 